\title{
Viscoelastic hinge formation in beams
}

\author{
A. Wineman and J. H. Min, Ann, Arbor, Michigan
}

(Received March 12, 1999)

\begin{abstract}
Summary. A constitutive equation for nonlinear viscoelasticity is used to model the mechanical response of solid polymers such as polycarbonate. The nonlinearity arises from a reduced time variable which causes stress relaxation to occur faster as strain increases. This constitutive equation is used to study the consequences of the interaction of the acceleration of stress relaxation with strain and the spatial variation of strain within the context of the structural theory of beams undergoing small displacements.

Two examples are presented - a cantilever beam under a concentrated force at the tip and a simply supported beam with an off center concentrated force. The regions with the greatest bending moments are also the regions of asccelerated stress relaxation. It is shown that there is a time after the transverse loads are applied when the stresses at the outer material elements in these cross sections decrease to such an extent that the location of the maximum stress begins to move to the interior of the cross section. In addition, the curvature begins to increase rapidly. The effect is analogous to that which occurs during the development of a plastic hinge in the context of elastic-perfectly plastic materials. These events can be interpreted as the onset and growth of a "viscoelastic hinge".
\end{abstract}

\section{Introduction}

There has been a great deal of interest in a particular class of constitutive equations for the nonlinear viscoelastic response of amorphous polymers, such as polycarbonate. The dominant feature of this class of constitutive equations is a reduced time variable by means of which stress relaxation occurs faster with increasing strain. This variable defines a relation between a material time scale and the laboratory time scale, and is often referred to as a "strain clock".

An important consequence of the "strain clock" is that there may not be a monotonic increase in stress under constant strain rate conditions. Instead, the stress can reach a local maximum, decrease to a local minimum and then increase. In addition, under constant stress rate conditions, the strain will increase slowly at first, and then, at a finite time, approach a very rapid rate of increase. In both cases, there is a time when the strain can begin to increase rapidly with respect to the stress. In the context of the response of polymers, this is referred to as yield. Shay and Caruthers [1], and Knauss and Emri [2], [3] provided numerical simulations which showed that such a constitutive equation can simulate yield in uniaxial constant strain rate tests. Wineman and Waldron [4] presented a mathematical analysis which showed that a "strain clock" based constitutive equation can describe yield under both uniaxial strain control and stress control histories. Wineman and Min [5] provided an analytical and numerical study of the predictions of this constitutive equation under biaxial yield for both constant strain rate and constant stress rate conditions.

Substantial experimental and theoretical effort is being directed at the evaluation of constitutive equations based on the clock concept. Shay and Caruthers [1], and Knauss and Emri 
[2], [3] investigated forms of the constitutive equation in which stress relaxation is accelerated by volumetric strain. McKenna and Zapas [6] obtained results which suggest that shear deformations also cause stress relaxation to be accelerated.

There has been a parallel analytical effort in which the constitutive equation is used to study the interaction of the acceleration of stress relaxation with strain and the spatial variation of strain. This effort has been motivated by experiments involving nonhomogeneous deformations as well as by structural applications in which polymeric materials may operate near yield. Most of the analytical work is restricted to conditions in which the strains and rotations are small while the material response is nonlinear. Moran and Knauss [7] studied the stresses near crack tips. Wineman and Waldron [4] considered circumferential shear of a hollow cylinder. In subsequent work, they discussed the same problem in the context of finite strains (see [8]). Wineman and Kolberg [9] provided an extensive discussion of pure bending. Wineman and Min [10], [11] discussed spherical and cylindrical containers under internal pressure.

The purpose of the present work is to study the consequences of the interaction of the acceleration of stress relaxation with strain and the spatial variation of strain within the structural theory of beams. In this case, strains vary both through the depth of a cross section and along the beam. When the material response can be described as linear viscoelastic, the regions with the greatest bending moments are also the regions with the greatest stresses and strains. When the material response is described by the constitutive equation with the "strain clock", these becomes regions of accelerated stress relaxation. It is shown that there is a time after transverse loads are applied to the beam when the stresses at the outer material elements in these cross sections begin to decrease and the location of the maximum stress begins to move to the interior of the cross section. In addition, the curvature begins to increase rapidly. The effect is analogous to that which occurs during the development of a plastic hinge in the context of elastic-perfectly plastic materials. These events are described here as the onset and growth of a "viscoelastic hinge".

The constitutive equation is introduced in Sect. 2. In Sect. 3, the equations are presented for two examples within the structural theory of bending - a cantilever beam under a concentrated force at the tip and a simply supported beam with an off center concentrated force. The formulation is restricted to conditions of small displacements. The numerical method of solution is outlined in Sect. 4, and results of numerical simulation are presented in Sect. 5. Final comments are presented in Sect. 6 .

\section{Nonlinear viscoelastic constitutive equation}

The displacement gradients are assumed to be sufficiently small so that the linearized straindisplacement relations are valid and can be used for structural analysis. Let $\sigma_{i j}$ and $\varepsilon_{i j}$ denote components of the stress and strain tensor, respectively, with respect to a Cartesian coordinate system. $\hat{\sigma}_{i j}=\sigma_{i j}-\left(\sigma_{k k} / 3\right) \delta_{i j}$ denotes the deviatoric part of the stress tensor, $\delta_{i j}$ denotes the Kronecker delta, and $\sigma_{k k} / 3$ denotes the hydrostatic part of the stress tensor, where repeated indices indicate summation. $\hat{\varepsilon}_{i j}=\varepsilon_{i j}-\left(\varepsilon_{k k} / 3\right) \delta_{i j}$ denotes the deviatoric part of the strain tensor, and $\varepsilon_{k k}=\theta$ is the volumetric strain or dilatation. It is assumed that $\varepsilon_{i j}=\sigma_{i j}=0$ for times less than zero. 
Then, the constitutive equation for isotropic linear viscoelastic response, in the regime of infinitesimal deformations, is

$$
\begin{gathered}
\hat{\sigma}_{i j}=2 \int_{0_{-}}^{t} \mu(t-s) \frac{\partial \hat{\varepsilon}_{i j}(s)}{\partial s} d s, \\
\sigma_{k k}=3 \int_{0_{-}}^{i} K(t-s) \frac{\partial \theta(s)}{\partial s} d s,
\end{gathered}
$$

where $\mu(t)$ is the shear relaxation function and $K(t)$ is the bulk relaxation function.

As in Knauss and Emri [2], [3], the constitutive equation for nonlinear viscoelastic response is taken as

$$
\begin{gathered}
\hat{\sigma}_{i j}=2 \int_{0_{-}}^{t} \mu[\xi(t)-\xi(s)] \frac{\partial \hat{\varepsilon}_{i j}(s)}{\partial s} d s, \\
\sigma_{k k}=3 \int_{0_{-}}^{t} K[\xi(t)-\xi(s)] \frac{\partial \theta(s)}{\partial s} d s,
\end{gathered}
$$

where $\xi(t)$ is defined in terms of the dilatation history $\theta(s), 0<s<t$, by the relation

$$
\xi(t)=\int_{0}^{t} \frac{d x}{\phi(\theta(x))} .
$$

$\xi(t)$ represents a reduced time (also referred to as pseudo, intrinsic, or material time) and introduces a nonlinear dependence on strain into the constitutive equations. $\phi$ has properties similar to those of the time-temperature shift function and is a monotonically decreasing function of $\theta$. In deformation histories for which $\theta(s)=\varepsilon_{k k}(s)$ increases with time, $\xi(t)$ increases faster than the physical time $t$. This results in an acceleration of stress relaxation. Wineman and Waldron [4] have shown that when the decrease of $\phi$ with $\theta$ is sufficiently large and rapid, the constitutive equation can simulate yield.

The form for $\phi$ used by Knauss and Emri [2], [3], and Wineman and Waldron [4] and also used here is the Dolittle shift function

$\log \phi=\frac{b}{2.303}\left(\frac{1}{f}-\frac{1}{f_{0}}\right)$

where $b$ is a material property. Knauss and Emri [2], [3] interpreted $f$ as the change in the fractional free volume in their general formulation. They related the change in the fractional free volume to the volumetric strain, i.e., $f=f(\theta)$, and assumed $f$ is linear in $\theta$. In this study, the form for $f$ is assumed to be

$f=f_{0}+c|\theta|$,

where $f_{0}$ denotes the fractional free volume at some reference state, and $c$ is a material constant that should be determined by experiment. When $c=0$, it follows from Eqs. (5) - (7) that $\xi(t)=t$, and Eqs. (3) and (4) reduce to those for linear viscoelasticity given by Eqs. (1) and 
(2). In an initial time interval, if $\theta(t)$ is sufficiently small, then, by Eqs. (5) $-(7), \xi(t) \approx t$ and the response described by Eqs. (3) and (4) approximates that given by Eqs. (1) and (2).

Note that Eq. (7) indicates dependence of $f$ on $|\theta|$ rather than $\theta$. Within this formulation, both volume increase and decrease accelerate stress relaxation. The response is then the same in tension and compression, which simplifies the presentation of the beam phenomenon which the subject of this paper.

For mathematical convenience, the constitutive equation is introduced in the form of Eqs. (3) and (4), which are expressed in terms of the shear and bulk moduli. It is well known for isotropic linear elasticity or viscoelasticity that the constitutive equation can be expressed in terms of alternate sets of two material properties, the choice being determined by the particular application under consideration. This paper is concerned with the structural theory of beams, in which each material element is primarily in a state of uniaxial extension or compression. It is thus useful to consider an alternate set of properties which is more appropriate to this state. Uniaxial extension in the $x_{1}$-direction is characterized by the conditions:

$\sigma_{22}(t)=\sigma_{33}(t)=0 \quad$ and $\quad \varepsilon_{22}(t)=\varepsilon_{33}(t)$.

Then, Eqs. (3) and (4) reduce to

$\sigma_{11}(t)=2 \int_{0_{-}}^{t} \mu[\xi(t)-\xi(s)] \frac{\partial}{\partial s}\left[\varepsilon_{11}(s)-\varepsilon_{22}(s)\right] d s$

and

$\sigma_{11}=3 \int_{0_{-}}^{t} K[\xi(t)-\xi(s)] \frac{\partial}{\partial s}\left[\varepsilon_{11}(s)+2 \varepsilon_{22}(s)\right] d s$.

In the case of linear elasticity, when $\mu$ and $K$ are constants, it is possible to eliminate $\varepsilon_{22}(s)$ from Eqs. (9) and (10) and express $\sigma_{11}(t)$ directly in terms of $\varepsilon_{11}(t)$ by means of an extensional modulus. This modulus is a rational polynomial in $\mu$ and $K$. For linear viscoelasticity, when $\xi(t)=t$, similar operations give an expression for $\sigma_{11}(t)$ in terms of the strain history $\varepsilon_{11}(s), 0 \leq s \leq t$, and an extensional relaxation property. This property is determined from $\mu(t)$ and $K(t)$ by solving a Volterra integral equation.

In the nonlinear case, there does not appear to be a direct analytical way of obtaining a relation between the stress $\sigma_{11}(t)$ and the strain history $\varepsilon_{11}(s), 0 \leq s \leq t$ from Eq. (9) and Eq. (10), and expressing the extensional property in terms of $\mu(t)$ and $K(t)$. However, it is reasonable to assume that such a relation may be possible. Thus, the constitutive equation for uniaxial extension is now taken as

$\sigma_{11}(t)=\varepsilon_{11}(0) G[\xi(t)]+\int_{0}^{t} G[\xi(t)-\xi(s)] \frac{\partial \varepsilon_{11}(s)}{\partial s} d s$,

in which $G(t)$ denotes the extensional relaxation function. Recall from Eq. (5), that the reduced time $\xi(t)$ depends on the dilatation, which is given by $\theta(t)=\varepsilon_{11}(t)+2 \varepsilon_{22}(t)$ in uniaxial extension. Thus, determination of $\sigma_{11}(t)$ also requires determination of $\theta(t)$ or $\varepsilon_{22}(t)$. Knauss and Emri [2], [3] determined the dilatation from stress by means of a bulk compliance function. Here, the dilatation is determined from the uniaxial strain by means of the Poisson's ratio function.

In a study of pure bending, Wineman and Kolberg [9] assumed that Poisson's ratio function was a constant. This implies that dilatation at a material element was proportional to 
$\varepsilon_{11}(s)$ and simplified the study. However, as the magnitude of the axial strain and dilatation increased, the shift function $\phi$ became very small, and $\xi(t)$ increased very rapidly. These effects are less severe when the Poisson's ratio function is time dependent.

From linear viscoelasticity, the dilatation function $\theta(t)=\varepsilon_{11}(t)+2 \varepsilon_{22}(t)$ can be expressed in terms of the Poisson's ratio function $\nu(t)$ by

$\theta(t)=\int_{0-}^{t}[1-2 \nu(t-s)] \frac{\partial \varepsilon_{11}(s)}{\partial s} d s$.

Consider a constant strain rate history, $\varepsilon_{11}(t)=\dot{\varepsilon}_{0} t$. Then, Eq. (12) becomes

$\theta(t)=\dot{\varepsilon}_{0} \int_{0}^{t}[1-2 \nu(s)] d s$

Note that $\dot{\theta}(t)=\dot{\varepsilon}_{0}(1-2 \nu(t))$. Poisson's ratio function for polymeric materials typically increases monotonically from $\nu(0) \approx 0.35$ to a value at large times of $\nu(\infty) \approx 0.499$ (Tschoegl [12], Staverman and Schwarzl [13]). For example, Knauss and Emri [2], [3] and Moran and Knauss [7] estimated $\nu(\infty) \approx 0.4999$ for PVAc. Thus, for large time, $1-2 \nu(\infty) \approx 0$, the rate of increase of $\theta(t)$ becomes very small, and $\theta(t)$ essentially approaches a limiting value. A similar restriction on the growth of $\theta(t)$ can be expected in the case of a general monotonically increasing strain history.

For this reason, the dilatation will be determined using the relation

$\theta(t)=\int_{0_{-}}^{t}[1-2 \nu(\xi(t)-\xi(s))] \frac{\partial \varepsilon_{11}(s)}{\partial s} d s$.

The constitutive equation for the material elements of the beam used in this paper now consists of Eqs. (11) and (14). It is intended that this constitutive equation represents the essential features of response of a generic material rather than of a specific material. It will be useful for bringing out a phenomenon which can be expected to occur to some extent in beams of all materials described by Eqs. (3) and (4).

\section{Bending-formulation}

The development of the structural theory of beams under transverse loading begins with a study of beam response under pure bending. Transversely loaded beams, whose ratio of depth to length is small, are regarded as assemblages of differential discs, each of which is in a state of pure bending. The formulation of the theory of pure bending of beams of nonlinear viscoelastic materials such as described in Sect. 2, and a thorough discussion of their response was provided by Wineman and Kolberg [9]. This formulation is summarized in the present section.

Consider a straight beam whose cross section has two lines of symmetry, which coincide with the $y$-and $z$-axes of a Cartesian coordinate system. Their origin is at the controid. Let the $x$-axis of the coordinate system be along the line which is normal to the cross section and which passes through its centroid. The assumptions which are used in the study of pure bending are that the deformation is sufficiently small that a change in the shape of the cross section may be neglected, and each plane section remains plane. If bending occurs in the $x-y$ plane, 
lines which are initially parallel to the center line ( $x$-axis) deform into circular arcs, and lines which are initially parallel to the $y$-axis remain straight and become radial line segments. At time $t$, the coordinate of the neutral axis relative to the centroid is denoted by $d(t)$ and its radius is denoted by $\varrho(t)$. Consider the line at coordinate $y$ which was initially parallel to the center line. The change in its distance from the $x$-axis due to the Poisson effect is neglected. The extensional strain of this line at time $t$ is obtained from a standard development and is given by

$\varepsilon(y, t)=[d(t)-y] \chi(t)$,

in which $\chi(t)=1 / \varrho(t)$ is the curvature.

By Eq. (11) the stress, $\sigma(y, t)$, on the area element of the cross section at the fixed coordinate $y$ is expressed in terms of its strain history, $\varepsilon(y, s), 0 \leq s \leq t$, by

$\sigma(y, t)=\varepsilon(y, 0) G[\xi(y, t)]+\int_{0}^{t} G[\xi(y, t)-\xi(y, s)] \frac{\partial}{\partial s} \varepsilon(y, s) d s$.

The reduced time $\xi(y, s)$ is given by

$\xi(y, s)=\int_{0}^{s} \frac{d \beta}{\phi(\theta(y, \beta))}$,

and, according to Eq. (14), the dilatation is determined from

$\theta(y, t)=\int_{0_{-}}^{t}[1-2 \nu(\xi(y, t)-\xi(y, s))] \frac{\partial \varepsilon_{11}(y, s)}{\partial s} d s$.

It is assumed that the resultant normal force on the cross section vanishes at each time $t$, that is

$F(t)=\iint_{A} \sigma(y, t) d A=0$,

in which the integration is taken over the undeformed shape of the cross section. Let $M(t)$ denote the bending moment on the cross section acting about the $z$-axis at time $t$. Then,

$M(t)=-\iint_{A} y \sigma(y, t) d A$.

Since the $z$-axis is an axis of symmetry of the cross section, and the material has the same response in tension and compression, it can be shown that $d(t)=0,0 \geq t$, so that the neutral axis coincides with the centroid at all times $t$. In this case, Eq. (15) reduces to

$\varepsilon(y, t)=-y x(t)$.

Equations (16) and (17) then imply that $\xi(y, s)=\xi(-y, s)$ and $\sigma(y, t)=-\sigma(-y, t)$. With the above assumptions Eq. (19) is satisfied.

Now consider a uniform beam under transverse loading. There is a shear force on each cross section, and the bending moment varies along the beam. Several assumptions are introduced in order to develop the structural theory of bending:

(i) the depth $h$ of the cross section is small compared to the length $L$;

(ii) the shear stress is small compared to the maximum normal stress, as in the linear theory, so that the pure bending formulation can be applied to each disc; 
(iii) the maximum deflection is small compared to the length $L$ of the beam. This allows the equilibrium equations to be applied in the reference configuration.

The strain and stress on the material element in the cross section at $x$ and at distance $y$ from the neutral axis can then be expressed as

$\varepsilon(x, y, t)=-y x(x, t)$,

$\sigma(x, y, t)=\varepsilon(x, y, 0) G[\xi(x, y, t)]+\int_{0}^{t} G[\xi(x, y, t)-\xi(x, y, s)] \frac{\partial}{\partial s} \varepsilon(x, y, s) d s$,

in which $x(x, t)=1 / \varrho(x, t)$, the curvature, can vary spatially with $x$ and with time $t$. The reduced time is given by

$\xi(x, y, s)=\int_{0}^{s} \frac{d \beta}{\phi(\theta(x, y, \beta))}$,

and the dilatation is determined from

$\theta(x, y, t)=\int_{0_{-}}^{t}[1-2 \nu(\xi(x, y, t)-\xi(x, y, s))] \frac{\partial \varepsilon_{11}(x, y, s)}{\partial s} d s$.

The condition that the resultant normal force on the cross section be zero requires

$\iint_{A} \sigma(x, y, t) d A=0$.

This condition is satisfied since the neutral axis coincides with the centroid. The resultant moment on the cross section is given by

$M(x, t)=-\iint_{A} y \sigma(x, y, t) d A$.

Nondimensional variables are introduced as follows:

$\bar{t}=t / \tau_{G}, \quad \bar{s}=s / \tau_{G}, \quad \bar{\xi}(\bar{x}, \bar{y}, \bar{t})=\xi(\bar{x}, \bar{y}, \bar{t}) / \tau_{G}$,

$\bar{x}=x / L, \quad \bar{y}=y / h, \quad \bar{h}=h / L$,

$\bar{\sigma}(\bar{x}, \bar{y}, \bar{t})=\sigma(\bar{x}, \bar{y}, \bar{t}) / G_{0}, \quad \bar{G}(\bar{t})=G(\bar{t}) / G_{0}, \quad \bar{x}(\bar{x}, \bar{t})=x(\bar{x}, \bar{t}) h$,

where $\tau_{G}$ is a characteristic relaxation time of $G(t)$ and $G_{0}=G(0)$.

With the above parameters, Eqs. (22)-(24) can be rewritten as

$\bar{\varepsilon}(\bar{x}, \bar{y}, \bar{t})=-\bar{y} \bar{x}(\bar{x}, \bar{t})$,

$\bar{\xi}(\bar{x}, \bar{y}, \bar{s})=\int_{0}^{\bar{s}} \frac{d \bar{\beta}}{\phi(\theta(\bar{x}, \bar{y}, \bar{\beta}))}$

and

$\bar{\sigma}(\bar{x}, \bar{y}, \bar{t})=-\bar{y} \bar{x}(\bar{x}, 0) \bar{G}[\bar{\xi}(\bar{x}, \bar{y}, \bar{t})]-\bar{y} \int_{0}^{\bar{t}} \bar{G}[\bar{\xi}(\bar{x}, \bar{y}, \bar{t})-\bar{\xi}(\bar{x}, \bar{y}, \bar{s})] \frac{\partial}{\partial \bar{s}} \bar{x}(\bar{x}, \bar{s}) d \bar{s}$.

Let $v(x, t)$ denote the vertical displacement at time $t$ of the point at $x$ on the neutral axis. In view of the above assumptions, the curvature $\varkappa(x, t)$ can be expressed in terms of the dis- 
placement $v(x, t)$ by

$x(x, t)=\frac{1}{\varrho(x, t)} \approx \frac{\partial}{\partial x}\left(\frac{\partial v(x, t)}{\partial x}\right)$.

Introducing the nondimensional vertical displacement, $\bar{v}(\bar{x}, \bar{t})=v(\bar{x}, \bar{t}) / L$, Eq. (32) reduces to

$\bar{\varkappa}(\bar{x}, \bar{t})=h \varkappa(\bar{x}, \bar{t})=\bar{h} \frac{\partial}{\partial \bar{x}}\left(\frac{\partial \bar{v}(\bar{x}, \bar{t})}{\partial \bar{x}}\right)$,

where $\tilde{h}$ is defined in Eq. (28)

\subsection{Cantilever beam}

Consider a uniform cantilever beam which is built in at $x=0$ and has length $L$. Its cross section is rectangular with width $b$ and height $h$. The beam is subjected to a concentrated force $P(t)$ at the free end $x=L$.

From moment balance, ignoring inertia, the bending moment at each section along the beam at time $t$ is given by

$M(x, t)=P(t)(L-x)=P(t) L(1-x / L)$.

With nondimensional variables introduced in Eq. (28), the moment-stress relation, Eq. (27), for the cantilever beam becomes

$M(\bar{x}, \bar{t})=-2 b h^{2} G_{0} \int_{0}^{1 / 2} \bar{y} \bar{\sigma}(\bar{x}, \bar{y}, \bar{t}) d \bar{y}$.

Finally, let Eq. (31) be substituted into Eq. (35) and then let both sides of the resulting equation be divided by $M_{0}=I G_{0} / h$, where $I=b h^{3} / 12$ is the moment of inertia of the undeformed rectangular cross section about the $z$-axis. Then, the nondimensional moment-curvature relation for the nonlinear vsicoelastic cantilever beam is given by

$$
\begin{aligned}
\bar{M}(\bar{x}, \bar{t}) & =\frac{M(\bar{x}, \bar{t}) h}{I G_{0}}=\frac{M(\bar{x}, \bar{t})}{M_{0}}=\frac{P(\bar{t}) L}{M_{0}}(1-\bar{x}), \\
& =24 \int_{0}^{1 / 2} \bar{y}^{2}\left\{\bar{x}(\bar{x}, 0) \bar{G}[\bar{\xi}(\bar{x}, \bar{y}, \bar{t})]+\int_{0}^{\bar{t}} \bar{G}[\bar{\xi}(\bar{x}, \bar{y}, \bar{t})-\bar{\xi}(\bar{x}, \bar{y}, \bar{s})] \frac{\partial}{\partial \bar{s}} \bar{x}(\bar{x}, \bar{s}) d \bar{s}\right\} d \bar{y} .
\end{aligned}
$$

When $P(\bar{t})$ is specified, this is an equation for $\bar{x}(\bar{x}, \bar{t})$.

Using the zero deflection and zero-slope boundary conditions on the vertical displacement at $x=0$ at each time $t$, the nondimensional curvature-deflection can be integrated to give

$\bar{h} \bar{v}(\bar{x}, \bar{t})=\int_{0}^{\bar{x}} \int_{0}^{\bar{x}_{1}} \bar{x}\left(\bar{x}_{2}, \bar{t}\right) d \bar{x}_{2} d \bar{x}_{1}$.

\subsection{Simply supported beam}

Consider a straight beam which is simply supported at its ends at $x=0$ and $x=L$. It is subjected to a concentrated force at the fixed location $x=e$, in which case the maximum deflection point need not coincide with the point where the force is applied. All assumptions introduced for the cantilever beam apply here also. 
Wineman and Kolberg [9] showed that because of the nonlinear viscoelasticity the response in pure bending depended very much on the shape of the cross section. For this reason, two different cross section shapes, a rectangular and an $I$-section, are considered for the simply supported beam. It will be seen that the beam response differs for the two cross section shapes.

From moment balance, ignoring inertia, the bending moment distribution for a simply supported beam along the beam length is given by

$$
\begin{aligned}
& M(\bar{x}, \bar{t})=P(\bar{t}) L(1-\bar{e}) \bar{x} \quad \text { for } \quad 0 \leq \bar{x} \leq \bar{e}, \\
& =P(\bar{t}) L(1-\bar{x}) \bar{e} \quad \text { for } \quad \bar{e} \leq \bar{x} \leq 1
\end{aligned}
$$

in which $\bar{e}=e / L$.

Consider a simply supported beam with a rectangular cross section and let the second moment of its area be denoted by $I_{R}$. Let Eqs. (22) and (23) be substituted into Eq. (27) and let the same non-dimensionalization be carried out as in the case of the cantilever beam, introducing the notation $M_{0 R}=I_{R} G_{0} / h$. The moment-curvature relation is given by

$$
\begin{aligned}
\bar{M}_{R}(\bar{x}, \bar{t}) & =\frac{M(\bar{x}, \bar{t})}{M_{0 R}}, \\
& =\frac{P(\bar{t}) L(1-\bar{e}) \bar{x}}{M_{0 R}} \quad \text { for } \quad 0 \leq \bar{x} \leq \bar{e} \\
& =\frac{P(\bar{t}) L \bar{e}(1-\bar{x})}{M_{0 R}} \quad \text { for } \quad \bar{e} \leq \bar{x} \leq 1 \\
& =24 \int_{0}^{1 / 2} \bar{y}^{2}\left\{\bar{x}(\bar{x}, 0) \bar{G}[\bar{\xi}(\bar{x}, \bar{y}, \bar{t})]+\int_{0}^{\bar{t}} \bar{G}[\bar{\xi}(\bar{x}, \bar{y}, \bar{t})-\bar{\xi}(\bar{x}, \bar{y}, \bar{s})] \frac{\partial}{\partial \bar{s}} \bar{x}(\bar{x}, \bar{s}) d \bar{s}\right\} d \bar{y}
\end{aligned}
$$

Next, consider the simply supported beam with an $I$-shaped cross section. The height of the $I$-section is $h$ and that of the web is $2 h_{1}$. The width of the flange is $b$ and the thickness of the web is $b_{1}$. Using the same nondimensional parameters as in the rectangular case and introducing additional parameters, $\bar{h}_{1}=h_{1} / h, \bar{b}_{1}=b_{1} / b$, the moment-curvature relation for the $I$-section can be obtained by integrating Eq. (27) separately over the web and flange. The result is

$$
M(\bar{x}, \bar{t})=-2 b h^{2} G_{0}\left[\bar{b}_{1} \int_{0}^{\bar{h}_{1}} \bar{y} \bar{\sigma}(\bar{x}, \bar{y}, \bar{t}) d \bar{y}+\int_{\bar{h}_{1}}^{1 / 2} \bar{y} \bar{\sigma}(\bar{x}, \bar{y}, \bar{t}) d \bar{y}\right] .
$$

Introduce a nondimensional moment $M_{0 I}=I_{I} G_{0} / h$, where

$$
I_{I}=\frac{b h^{3}-8\left(b-b_{1}\right)}{12} \underline{h_{1}^{3}}
$$

denotes the second moment of the area of the $I$-section. Then the nondimensional moment for the $I$-shaped simply supported beam is given by

$$
\begin{aligned}
\bar{M}_{I}(\bar{x}, \bar{t}) & =\frac{M(\bar{x}, \bar{t})}{M_{0 I}}, \\
& =\frac{P(\bar{t}) L(1-\bar{e}) \bar{x}}{M_{0 I}} \quad \text { for } \quad 0 \leq \bar{x} \leq \bar{e}, \\
& =\frac{P(\bar{t}) L \bar{e}(1-\bar{x})}{M_{0 I}} \quad \text { for } \quad \bar{e} \leq \bar{x} \leq 1 .
\end{aligned}
$$


Substituting Eq. (31) into Eq. (40), the nondimensional moment-curvature relation for the $I$-beam becomes

$$
\begin{aligned}
\bar{M}_{I}(\bar{x}, \bar{t})= & \frac{M(\bar{x}, \bar{t})}{M_{0 I}} \\
= & 24 I^{*} \int_{\bar{b}_{1}} \int_{0}^{\bar{h}_{1}} \bar{y}^{2}\left[\bar{x}(\bar{x}, 0) \bar{G}[\bar{\xi}(\bar{x}, \bar{y}, \bar{t})]+\int_{0}^{\bar{t}} \bar{G}[\bar{\xi}(\bar{x}, \bar{y}, \bar{t})-\bar{\xi}(\bar{x}, \bar{y}, \bar{s})] \frac{\partial}{\partial \bar{s}} \bar{x}(\bar{x}, \bar{s}) d \bar{s}\right] d \bar{y} \\
& \left.+\int_{\bar{h}_{1}}^{1 / 2} \bar{y}^{2}\left[\bar{x}(\bar{x}, 0) \bar{G}[\bar{\xi}(\bar{x}, \bar{y}, \bar{t})]+\int_{0}^{\bar{t}} \bar{G}[\bar{\xi}(\bar{x}, \bar{y}, \bar{t})-\bar{\xi}(\bar{x}, \bar{y}, \bar{s})] \frac{\partial}{\partial \bar{s}} \bar{x}(\bar{x}, \bar{s}) d \bar{s}\right] d \bar{y}\right\}
\end{aligned}
$$

in which

$$
I^{*}=\frac{\frac{b h^{3}}{12}}{\frac{b h^{3}-8\left(b-b_{1}\right) h_{1}^{3}}{12}}=\frac{1}{1-8\left(1-\bar{b}_{1}\right) \bar{h}_{1}^{3}} .
$$

Using the zero-deflection boundary conditions on the vertical displacement at $x=0$ and $x=L$ at each time $t$, the nondimensional curvature-deflection can be integrated to give the nondimensional spatially varying deflection at time $t$,

$\bar{h} \bar{v}(\bar{x}, \bar{t})=\int_{0}^{\bar{x}} \int_{0}^{\bar{x}_{1}} \bar{x}\left(\bar{x}_{2}, \bar{t}\right) d \bar{x}_{2} d \bar{x}_{1}-\bar{x} \int_{0}^{1} \int_{0}^{\bar{x}_{1}} \bar{x}\left(\bar{x}_{2}, \bar{t}\right) d \bar{x}_{2} d \bar{x}_{1}$.

When a force $P(\bar{t})$ is specified, the nondimensional curvature, $\bar{x}(\bar{x}, \bar{t})$, can be evaluated from Eq. (39) for rectangular section or Eq. (43) for $I$-section. Then, the deflection $\bar{v}(\bar{x}, \bar{t})$ can be computed by Eq. (45).

\section{Numerical method of solution}

Because of the nonlinearity introduced into the moment-curvature relation by the reduced time, numerical methods are used to determine the stress distribution and deflection histories of the beam. The governing equations involve integrations over three variables: the nondimensional time $\bar{t}, \bar{y}$ over the cross section, and $\bar{x}$ along the beam length. The method of numerical integration over each variable is outlined here. A detailed discussion can be found in [14].

Solutions are obtained at an equally spaced set of times $\bar{t}_{i}, i=0,1,2, \ldots, n-1, n$. The reduced time integral is approximated using the trapezoidal rule. If $\bar{\xi}\left(t_{n-1}\right)$ has been evaluated, the trapezoidal rule is then readily used to obtain the updated value $\bar{\xi}\left(\bar{t}_{n}\right)$. The Volterra convolution integral operator

$$
\int_{\bar{t}_{0}}^{\bar{t}_{n}} G\left[\bar{\xi}\left(\overline{t_{n}}\right)-\bar{\xi}(s)\right] \frac{\partial}{\partial s} \varepsilon(s) d s
$$

is also approximated using the trapezoidal rule. In addition, it is assumed that the relaxation function $G(\bar{t})$ is expressed as a sum of exponentials. This enables a recurrence relation to be 
developed which is used to update the Volterra convolution integral operator from its value at $\bar{t}_{n-1}$ to its value at $\bar{t}_{n}$. These updating methods substantially reduce the computation time. A simple finite difference expression is used for the time derivative of strain.

Integration with respect to the cross section variable $\bar{y}$ occurs in the moment-curvature relations (36) for the rectangular cross section and (43) for the $I$-section. The Simpson's $3 / 8$ rule is used for this integration because it provides greater accuracy than the trapezoidal rule and requires fewer subintervals.

The deflection is obtained by integrating the curvature $\bar{x}(\bar{x}, \bar{t})$ with respect to the beam length variable $\bar{x}$, as shown in Eq. (37) for the cantilever beam and (45) for the simply supported beam. These are first reduced to two successive single integrals. For example, Eq. (37) becomes

$\bar{h} \bar{v}(\bar{x}, \bar{t})=\int_{0}^{\bar{x}} \varkappa^{*}\left(x_{1}, \bar{t}\right) d x_{1}, \quad \varkappa^{*}\left(x_{1}, \bar{t}\right)=\int_{0}^{x_{1}} \bar{x}\left(x_{2}, \bar{t}\right) d x_{2}$.

The trapezoidal rule is then used to approximate these integrals on a discrete set of equally spaced positions along the beam.

The system of equations to be considered consists of the dilatation-curvature relation (25), the moment-curvature relation (36), (39) or (43) and the deflection-curvature relation (37) or (45). Under load control conditions, when the force history is specified, the dilatation-curvature relation and the appropriate moment-curvature relation are solved for the curvature history, and then the deflection history is evaluated. Under deflection control conditions, when the deflection history is specified, the system to be solved also includes the deflectioncurvature relation.

First, consider load control in which a concentrated force history $P(\bar{t})$ is specified. Then the moment distribution history $\bar{M}(\bar{x}, \bar{t})$ is known. Suppose $\bar{x}\left(\bar{x}, \bar{t}_{k}\right)$ and $\theta\left(\bar{x}, \bar{y}, \bar{t}_{k}\right)$ have been found at previous times $\bar{t}_{k}<\bar{t}_{n}$. Let $\bar{x}\left(\bar{x}, \bar{t}_{n}\right)$ be assumed by extrapolation from solutions found at earlier times. The discretized dilatation-curvature relation is solved for $\theta\left(\bar{x}, \bar{y}, \bar{t}_{n}\right)$. These are then used to evaluate the right-hand sides of the discretized moment-curvature relation (36), (39) or (43). The result is compared with $\bar{M}\left(\bar{x}, \bar{t}_{n}\right)$. The Newton-Raphson method is used to modify $\bar{x}\left(\bar{x}, \bar{t}_{n}\right)$ and the process is repeated until the right-hand side of the appropriate discretized moment-curvature relation is sufficiently close to the specified moment $\bar{M}\left(\bar{x}, \bar{t}_{n}\right)$.

Next, consider deflection control. Suppose that the maximum deflection at each time is specified. In this case, the corresponding concentrated force history $P(\bar{t})$ is estimated and the preceding process is used to determine the curvature history. The deflection history is computed. A method utilizing Newton-Raphson iteration is then used modify $P(\bar{t})$ until the computed deflection is sufficiently close to the specified deflection.

The increments $\Delta \bar{t}$ in the nondimensional time, $\Delta \bar{y}$ in the cross section, and $\Delta \bar{x}$ along the beam were determined by considering relative errors for the case of a cantilever beam under load control with $P(\bar{t}) L / M_{0}=\left(\alpha_{1} L / M_{0}\right) \bar{t}=0.4 \bar{t}$. The curvature at the clamped end $(x / L=0.0)$ of the cantilever beam is greater than that at any other point along the beam. Therefore, the curvature at the clamped end of the beam is used to estimate the relative errors corresponding to the choices of $\Delta \bar{t}$ and $\Delta \bar{y}$. For the estimate of the relative error due to the choice of $\Delta \bar{x}$, the deflection at the tip $(x / L=1.00)$ of the beam is considered.

Consideration of the competition between accuracy and cost in computations led to the selection of $\Delta \bar{t}=0.00001, \Delta \bar{y}=0.02$ and $\Delta \bar{x}=0.025$. The relative error between the curvature at the clamped end of the beam at $\bar{t}=0.1$ calculated using this time step and that calculated using the next smaller time step is less than $0.42 \%$. The relative error between the 
curvature at the clamped end of the beam at $\bar{t}=0.1$ calculated using this choice of $\Delta \bar{y}$ and that calculated using the next smaller choice is less than $0.01 \%$. The relative error between the tip deflection at $\bar{t}=0.1$ calculated using this choice of $\Delta \bar{x}$ and that calculated using the next smaller choice is less than $0.01 \%$. Further details can be found in [14].

The numerical method requires that the dilatation be solved at each nodal point $\bar{y}_{i}$ in the cross section, each nodal point $\bar{x}_{j}$ along the beam and at each time $\bar{t}_{k}$. In order to reduce computation time, an approximation was used to evaluate the dilatation over the cross section. In the linear viscoelastic case, Eq. (21) and Eq. (12) show that the dilatation varies linearly over the cross section. The dilatation $\theta(\bar{x}, \bar{y}, \bar{t})$ is also assumed to vary linearly over the cross section in the nonlinear viscoelastic case. The variation will be approximately linear for small times, before the curvature or bending strains become very large. For larger times, this approximation may become less accurate. But as the maximum strains occur at the outer material elements of the beam, the dilatation will be larger there, and the assumption accounts for this. Also, the purpose here is not to describe the response of a particular material, but to describe a phenomenon which can occur in beams composed of materials represented by the constitutive theory in Sect. 2. The assumption will enable this phenomenon to be discussed, but with reduced computation time.

Thus, the dilatation in the NLVE constitutive equation is represented by

$\theta(\bar{x}, \bar{y}, \bar{t})=-y \theta^{*}(\bar{x}, \bar{t})$,

in which $\theta^{*}(\bar{x}, \bar{t})$ is expressed as

$\theta^{*}(\bar{x}, \bar{t})=\int_{0}^{\bar{t}}[1-2 \nu[\bar{\xi}(\bar{x}, \bar{t})-\bar{\xi}(\bar{x}, \bar{s})]] \frac{\partial \bar{x}(\bar{x}, \bar{s})}{\partial \bar{s}} d \bar{s}$,

where

$\bar{\xi}(\bar{x}, \bar{t})=\int_{0}^{\bar{t}} \frac{d \bar{\beta}}{\phi\left(\theta^{*}(\bar{x}, \bar{\beta})\right)}$.

The material property functions $G(t)$ and $\nu(t)$ were chosen to represent properties of a generic material rather than of a specific material. In this way, the results of the present study can bring out features which can be expected to occur to some extent in all materials of the current class under consideration.

For the purpose of numerical studies, single exponential representations were used for $G(t)$ and $\nu(t)$,

$G(t)=G_{0}+\left(G_{\infty}-G_{0}\right)\left(1-e^{-t / \tau_{G}}\right)$,

$\nu(t)=\nu_{0}+\left(\nu_{\infty}-\nu_{0}\right)\left(1-e^{-t / \tau_{\nu}}\right)$,

in which $G_{0}$ and $\nu_{0}$ denote initial values of the extension modulus and the Poisson's ratio function, $G_{\infty}$ and $\nu_{\infty}$ denote the values as $t$ becomes large, and $\tau_{G}$ and $\tau_{\nu}$ denote the characteristic times for the extensional modulus and the Poisson's ratio function, respectively. The characteristic relaxation time $\tau_{G}$ used to define nondimensional quantities in Eq. (28) is chosen to be the characteristic time appearing in Eq. (50). Introducing the nondimensional $\bar{t}=t / \tau_{G}$ in Eq. (51) leads to the nondimensional time parameter $\tau^{*}=\tau_{\nu} / \tau_{G}$.

The free volume parameters, $c=1.00, b=0.16$, and $f_{0}=0.01$, the parameters for the extensional relaxation modulus $G_{0}=1791.6 \mathrm{MPa}$ and $G_{\infty}=0.6023 \mathrm{MPa}$, and the parameters for the Poisson's function, $\nu_{0}=0.4125$ and $\nu_{\infty}=0,4999$, are chosen to be the same as used by Knauss and Emri [2], [3] for PVAc. 
A discussion of the influence of the nondimensionalized time parameter $\tau^{*}$ was presented in [14]. The dilatation history at the top element at the clamped end of the cantilever beam was studied under loading described earlier. The dilatation histories approached a limiting value as the material response entered the incompressible region due to the reduced time scale. A value of $\tau^{*}=20$ was chosen as reasonable to bring out the effects of material nonlinearity whithin the time interval of $\bar{t}=0.1$.

\section{Numerical results}

The cantilever beam under load control is considered first. It is assumed that the concentrated force $P(\bar{t})$ at the beam tip $\bar{x}=x / L=1$ increases at a constant rate, i.e., $P(\bar{t})=\alpha_{1} \bar{t}$ where $\alpha_{1}$ is a constant. Then, $P(\bar{t}) L / M_{0}=\left(\alpha_{1} L / M_{0}\right) \bar{t}$ in Eq. (36). The value $\alpha_{1} L / M_{0}=0.4$ was used in the numerical simulation and simulation was carried out to $\bar{t}=0.1$ as a reasonable choice based on the cost of computation.

The curvature histories at the clamped end for linear viscoelastic (LVE) and nonlinear viscoelastic (NLVE) response are shown in Fig. 1. The curvature distributions along the beam length at the final simulation time $\bar{t}=0.1$ for linear and nonlinear viscoelastic response are shown in Fig. 2. For small times, the curvatures for LVE and NLVE are very close. However, as $\bar{t}$ increases, the NLVE response begins to increase sharply relative to that for the LVE case. This is due to the rapid increase of the material or reduced time relative to the real time $\bar{t}$, which produces more stress relaxation.

Consider the cross section at an arbitrary position along the beam. During the initial time interval when the LVE and NLVE responses are very close, the outer material elements of the cross section carry higher stress and thus contribute more to the bending moment on the cross section than do those closer to the neutral axis. However, as $\bar{t}$ increases, the strains and dilatation at the outer material elements increase faster than at those closer to the neutral axis. The acceleration of stress relaxation at the outer material elements for NLVE causes their stresses and thus their contribution to the bending moment to diminish rapidly. In order to compen-

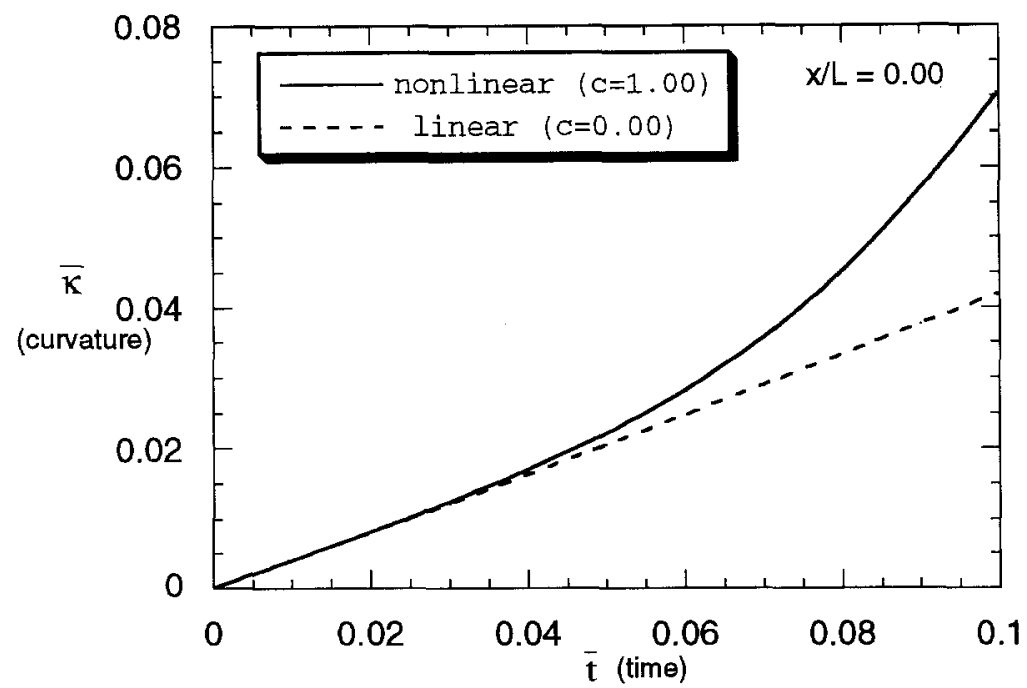

Fig. 1. Curvature histories at the clamped end of a cantilever beam under load control, for LVE $(c=0.0)$ and NLVE $(c=1.0)$ 


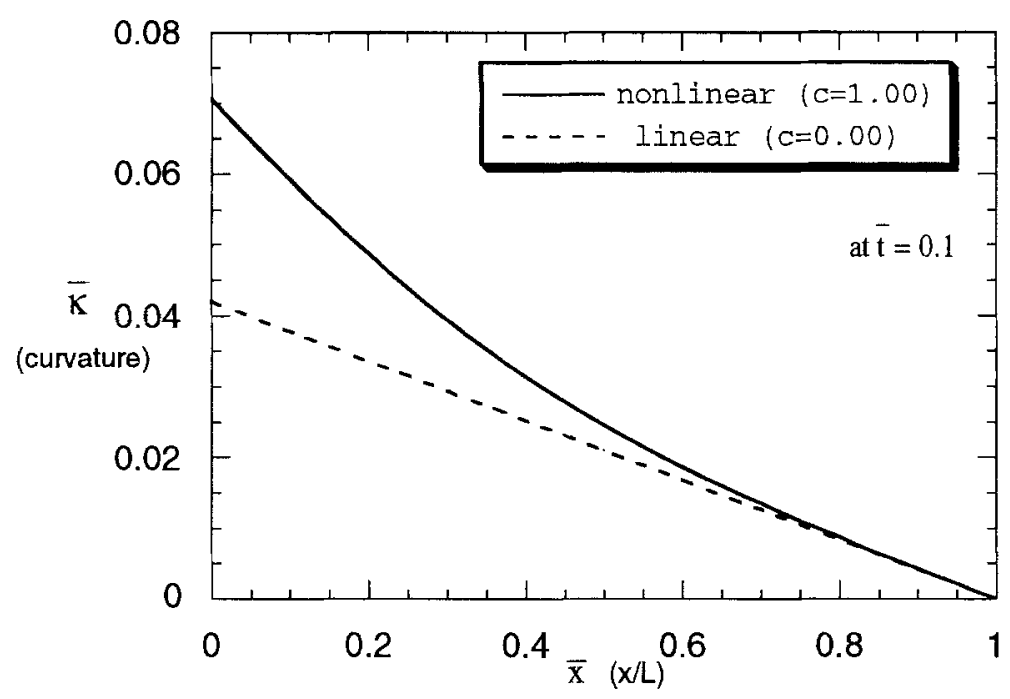

Fig. 2. Curvature distributions along the beam length of a cantilever beam under load control at the final simulation time $\bar{t}=0.1$, for $\operatorname{LVE}(c=0.0)$ and $\operatorname{NLVE}(c=1.0)$

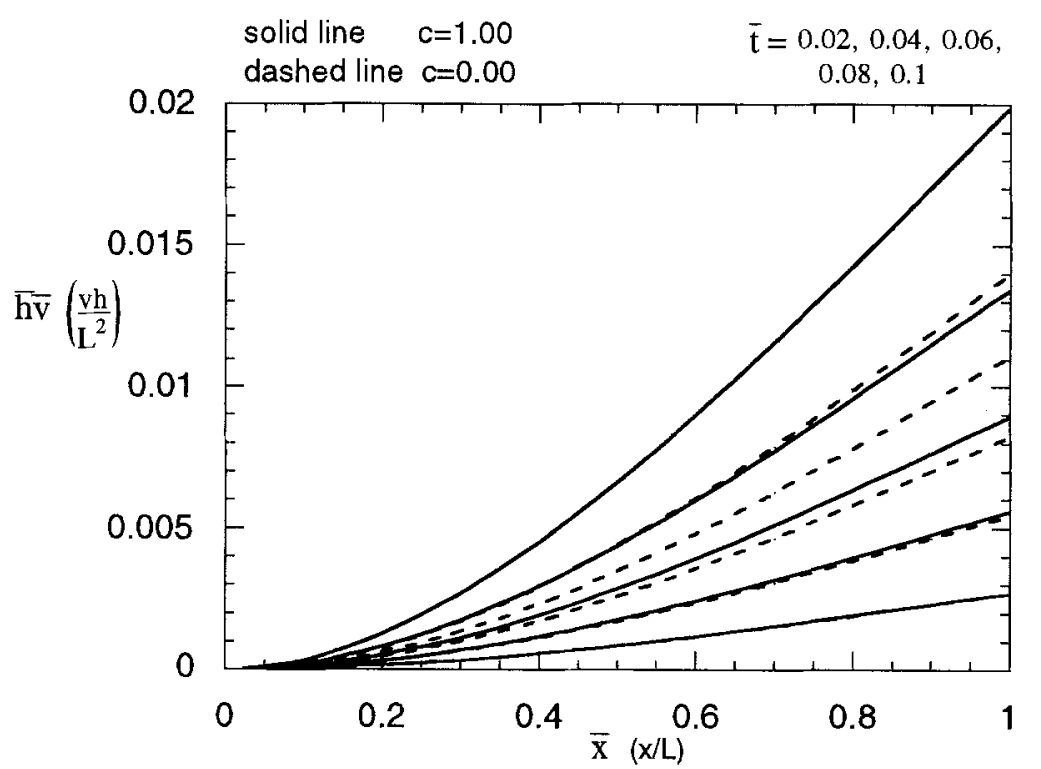

Fig. 3. Deflection profiles along the beam length for various times of a cantilever beam under load control, for LVE $(c=0.0)$ and NLVE $(c=1.0)$

sate for this, the curvature in the NLVE case begins to increase faster than that in the LVE case so that the stresses closer to the neutral axis increase and contribute more to the bending moment. This process occurs faster at the cross section near the clamped end where there is a bigger moment, and results in a sharp increase of curvature.

The nondimensional deflections along the beam for LVE and NLVE are shown in Fig. 3. For small times, there is no significant difference between the deflections for LVE and NLVE. As indicated in Figs. 1 and 2, the curvature at sections near the clamped end increases much faster in the NLVE case than in the LVE case. This causes the tip deflection in the NLVE case to increase faster than in the LVE case. Also note that deflection was limited to a maximum 


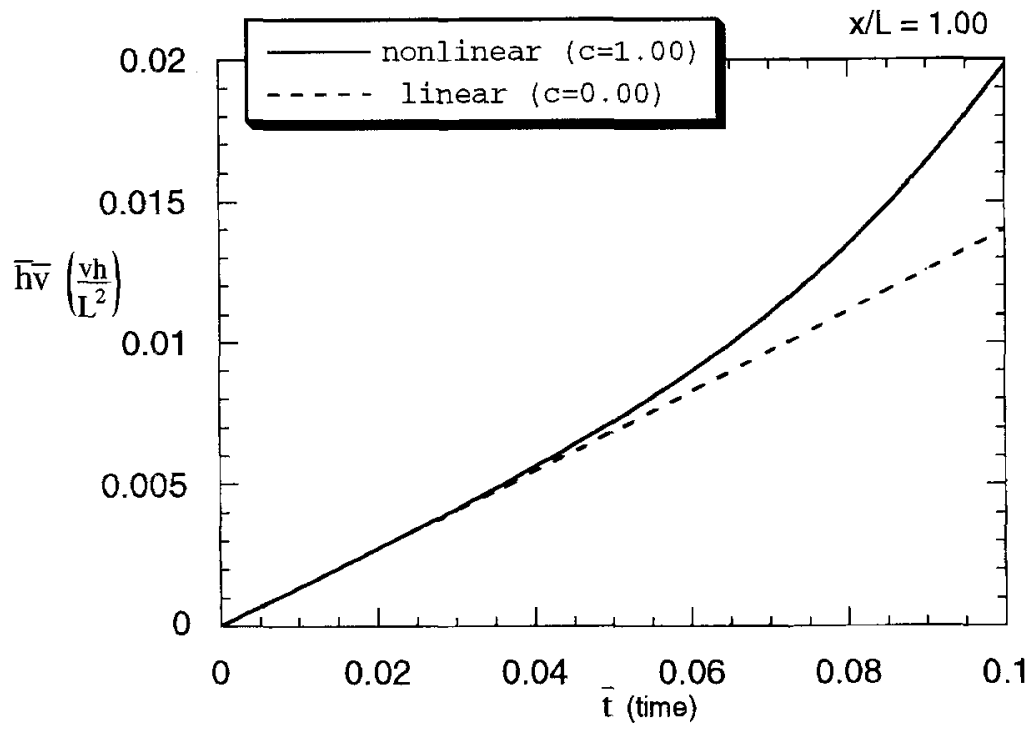

Fig. 4. Deflection histories at the tip $(x / L=1.00)$ of a cantilever beam under load control, for LVE $(c=0.0)$ and NLVE $(c=1.0)$

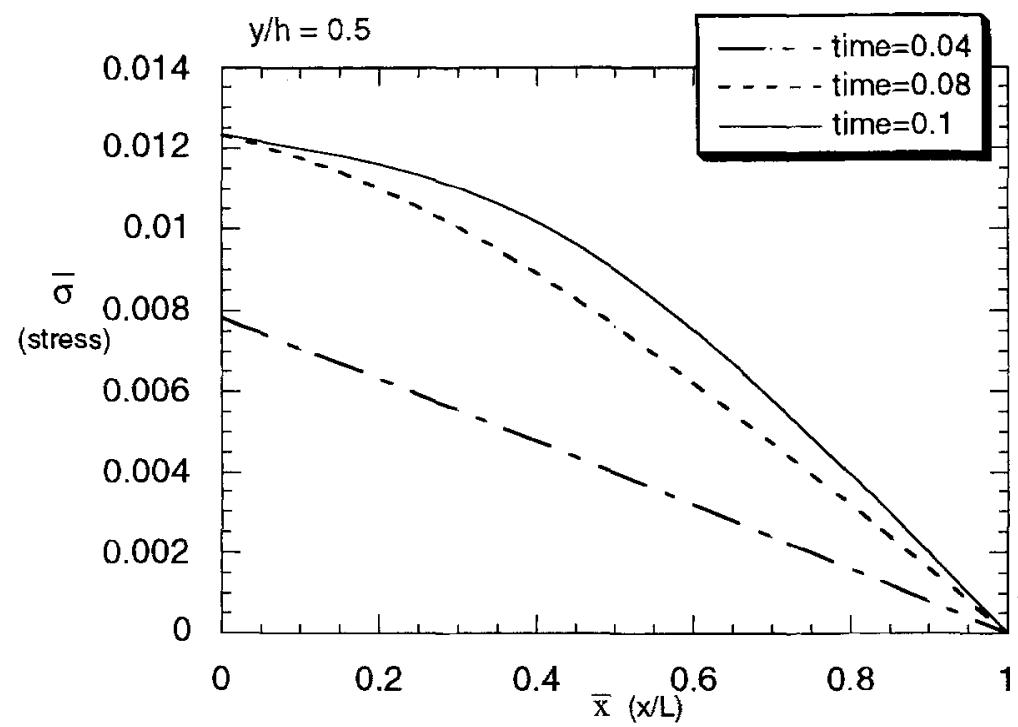

Fig. 5. Stresses at the top element of the cross section along a cantilever beam under load control, for various times

value of 0.02 in this calculation. This value is near the maximum acceptable value consistent with the small displacement approximation. Figure 4 shows the LVE and NLVE deflection histories at the tip, $\vec{x}=1$, and indicates the consequence of the accelerated stress relaxation for NLVE. At the final simulation time $\bar{t}=0.1$, the deflection for NLVE is more than $40 \%$ larger than that for LVE.

Figure 5 shows the stress at the element at the top of each cross section $(\bar{y}=0.5)$ at various times and illustrates the consequences of the acceleration of stress relaxation along the beam length. Up to time $\bar{t}=0.04$, there has been no noticeable acceleration of stress relaxation, and the NLVE response differs little from the LVE response. However, as time increases, 


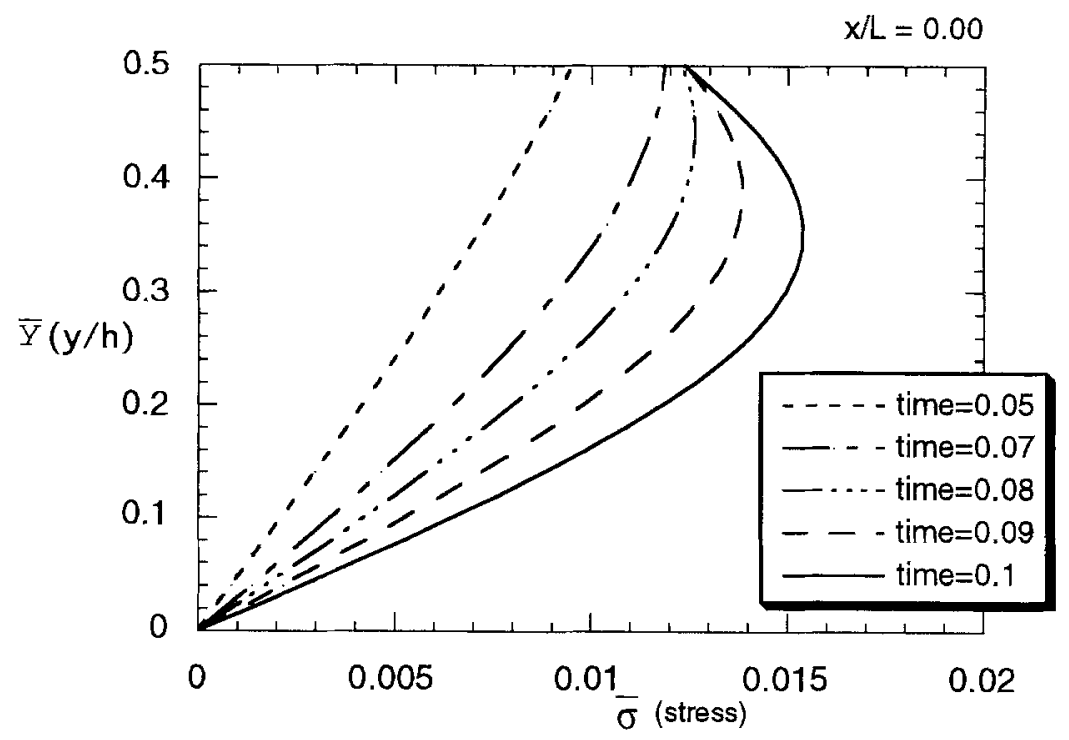

Fig. 6. Stress distributions through the beam thickness at the clamped end of a cantilever beam under load control, for various simulation times

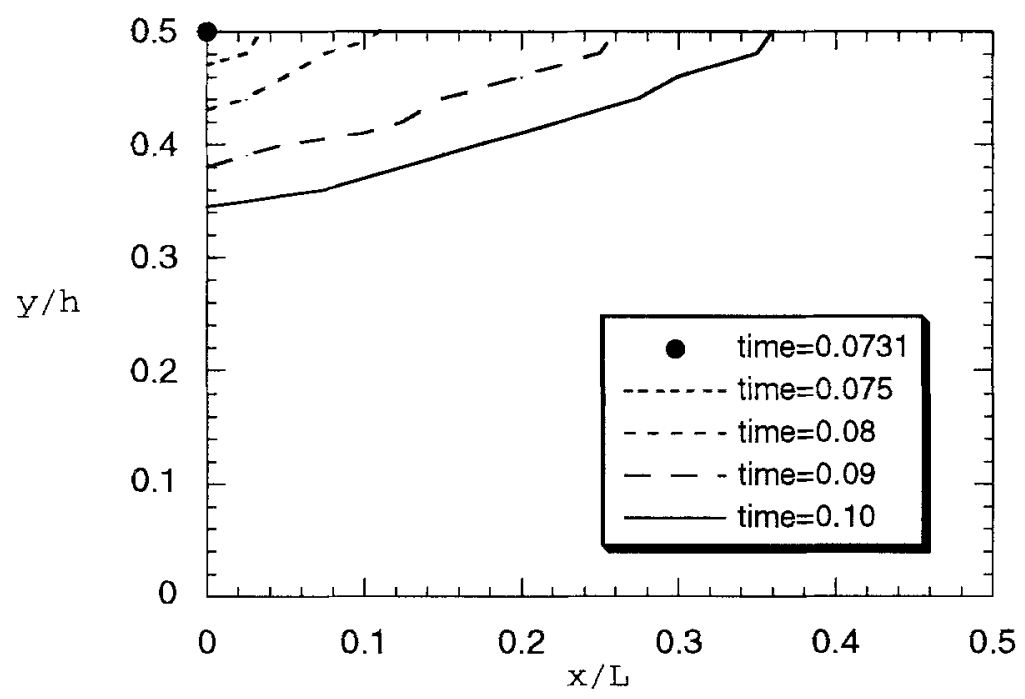

Fig. 7. Growth of stress relaxation zones with time in a cantilever beam under load control

the reduced time effects become significant so that stress relaxation is accelerated. At the clamped end, where the bending moment is largest, the stress at $\bar{t}=0.1$ has decreased so that it almost coincides with the stress at $\bar{t}=0.08$. This shows that faster relaxation occurs at the section with the higher moment.

Another view of this is seen in Fig. 6, which shows stress distributions over the cross section at $\bar{x}=0.0$ at various times. For $\bar{t}>0.07$, the location of the maximum stress is no longer at the outer most material element, but has moved closer to the neutral axis. In the outer part of the cross section, there is the onset and growth of a zone of decreased stress and hence decreased contribution to the moment transmitting capability of the beam. Similar zones develop in adjacent cross sections. 
To study this phenomenon further, the location $\bar{y}^{*}<0.5$ within each cross section where stress is a maximum was determined at a set of times. The times $\bar{t}$ and cross sections $\bar{x}$, where $\bar{y}^{*}<0.5$ indicate zones of accelerated stress relaxation and are shown in Fig. 7. These zones are initiated at time $\bar{t}=0.0731$ at the clamped end, when the stress at the outer material element $\bar{y}=0.5$ decreases faster than at an adjacent interior element at, say, $\bar{y}=0.48$. At the final simulation time $\bar{t}=0.1$, the zone extends to $\bar{y}^{*} \approx 0.345$ at the clamped end and to the outer material element at $\bar{x}=0.36$ along the beam. Thus, consider the solid line in Fig. 7 , which corresponds to time $\bar{t}=0.1$. At each cross section, the stresses on the material elements above the solid line are less than those at the solid line. The stress distributions decribed here are analogous to those which occur during the formation of yield zones in the response of elastic-perfectly plastic beams. In the present context, this zone of accelerated stress-relaxation can be thought of as a viscoelastic yield zone. Moreover, the increased curvature is analogous to that which occurs in the formation of plastic hinges. The process can thus be described as the onset and evolution of a viscoelastic hinge.

Now consider the same cantilever beam, but under deflection control. It is assumed that the deflection history at the tip, $\bar{x}=x / L=1$, has a nondimensionalized constant rate form, i.e., $\bar{h} \bar{v}(\bar{x}, \bar{t})=\beta_{1} \bar{t}$ in Eq. (3.23), where $\beta_{1}$ is a constant. $\beta_{1}=2.5$ is chosen with the maximum numerical nondimensionalized simulation time $\bar{t}=0.01$.

Figure 8 shows curvature histories for NLVE at various sections, $\bar{x}=0.00,0.20,0.50$, along the beam length. For LVE, the curvature is given by expressions of the form $x=f(\bar{x}) g(\bar{t})$, that is, the curvature-time plots have similar shapes, but differ by a scale factor which depends on $\bar{x}$. The curvatures in the NLVE case are different. The curvature history at $\bar{x}=0.2$ is almost linear in $\bar{t}$, while the slope of $\bar{x}$ at $x=0.0$ increases with time $\bar{t}$ and the slope of $\bar{x}$ at $\bar{x}=0.5$ decreases with time $\bar{t}$.

Another view of this is shown in Fig. 9, which represents the curvature profiles along the beam for NLVE and LVE at the final simulation time, $\bar{t}=0.01$. For NLVE, the curvatures near the clamped end, up to $\bar{x}=0.2$, are higher and the curvatures beyond $\bar{x}=0.2$ are less than those for LVE. A region of rapidly increasing curvature develops near $\bar{x}=0.0$ due to the acceleration of stress relaxation. The curvature in this region for NLVE contributes more to the tip deflection than for the LVE. This can be interpreted as the formation of a viscoelastic "hinge" just as in the load control case.

Figure 10 shows the history of the concentrated force at the beam tip. Its slope decreases with time, which reduces the rate of increase of the moment and the corresponding curvature near the support. Otherwise, due to the acceleration of sress-relaxation and creep, the tip deflection would increase too rapidly. This response is similar to that found in circular shear of a hollow cylinder (Wineman and Waldron [4]). In this case, a hollow cylinder is bonded to a fixed support at its inner surface and the outer surface is subjected to a constant rate of rotation about the central axis. The material near the inner surface develops a zone of large shear strain due to accelerated stress relaxation. This zone contributes to a rapid increase of rotation at the outer surface. The moment applied to the outer surface must be rapidly reduced in order to control the rotation.

A study of the stress distribution history can be found in [14], but is omitted for the sake of brevity. The results indicate the onset and growth of a zone of the acceleration of stress relaxation as in the load control case.

Next, consider a simply supported beam which has a concentrated force applied at $x / L=0.70$. It is assumed that $P(\bar{t})=\gamma \bar{t}$ where $\gamma$ denotes a constant. Both rectangular and $I$ shaped cross sections are discussed. The same nondimensionalized load is applied for the cases of both the rectangular and $I$-sections. Calculations were carried out for $\left(\gamma L / M_{0 R}\right) \bar{t}$ 


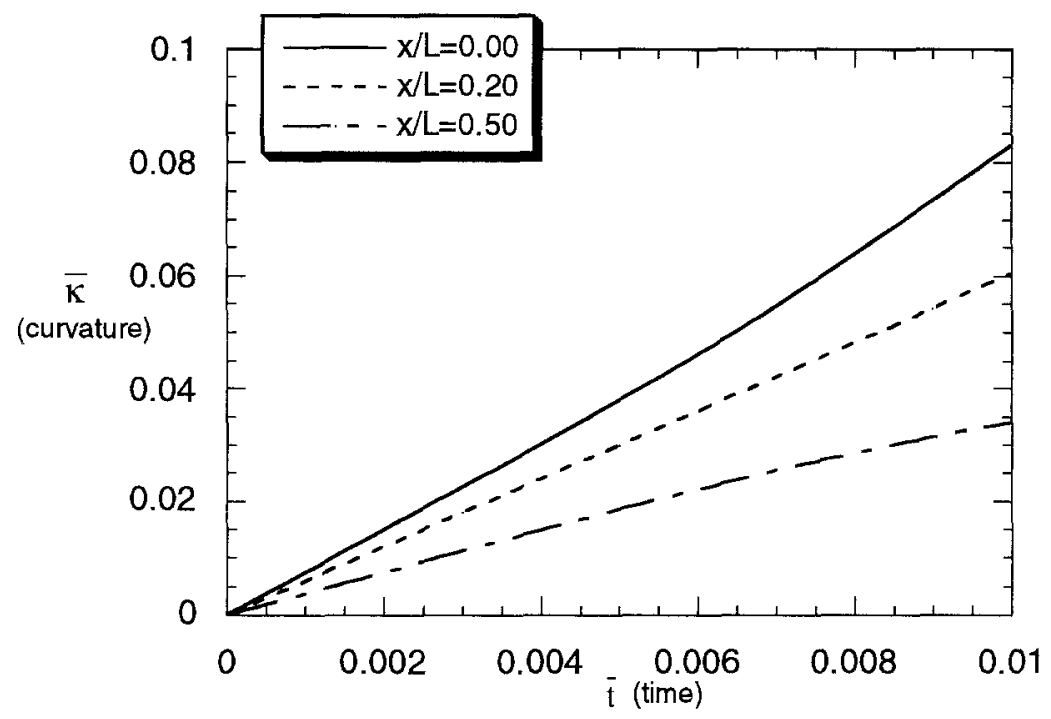

Fig. 8. Curvature histories for various positions along the beam length of a cantilever beam under deflection control

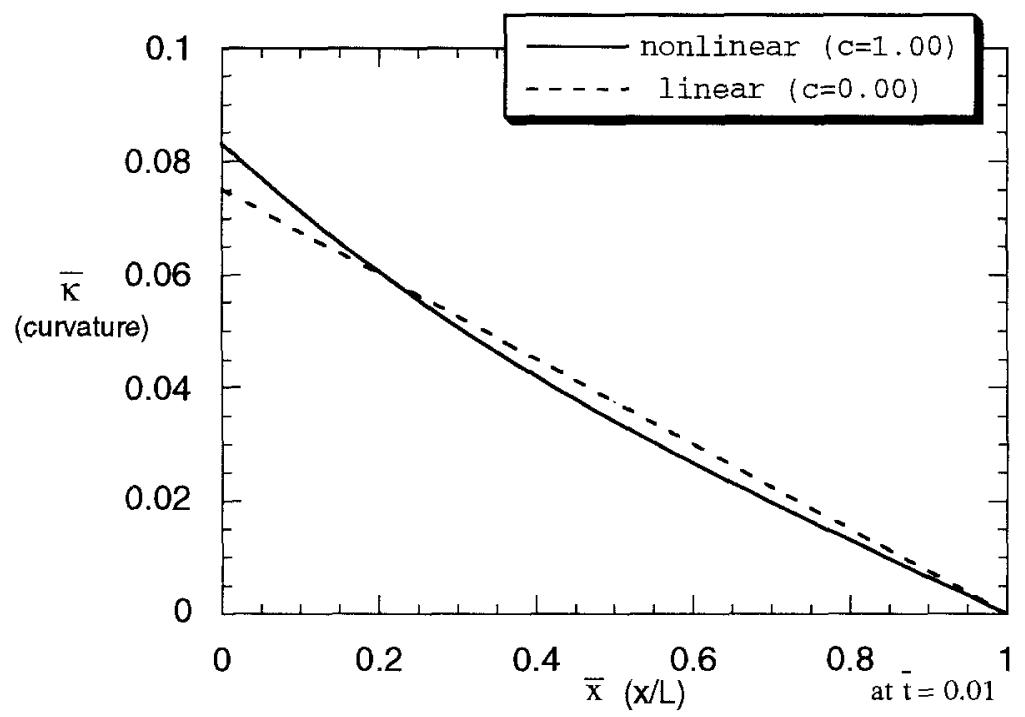

Fig. 9. Curvature distributions along a cantilever beam under deflection control at the final simulation time $\bar{t}=0.01$

$=\left(\gamma L / M_{0 I}\right) \bar{t}=2.0 \bar{t}$ until the final nondimensionalized time $\bar{t}=0.1$. For the $I$-section, $\bar{h}_{1}=0.44$ and $\bar{b}=0.2$.

Curvature distributions along the beam at the final simulation time are presented in Fig. 11. At each section, the curvature for the $I$-beam is greater than that for the rectangular beam, and both are greater than for LVE. These results are consistent with previous results (Kolberg and Wineman [9]) which show that the $I$-section rapidly becomes ineffective in transmitting bending moments due to the acceleration of stress relaxation in the flange. Note that there is a zone near $\bar{x}=0.7$, where the curvature in the NLVE case is large compared to that in the LVE case. Figure 12 shows curvature distributions along the beam for 


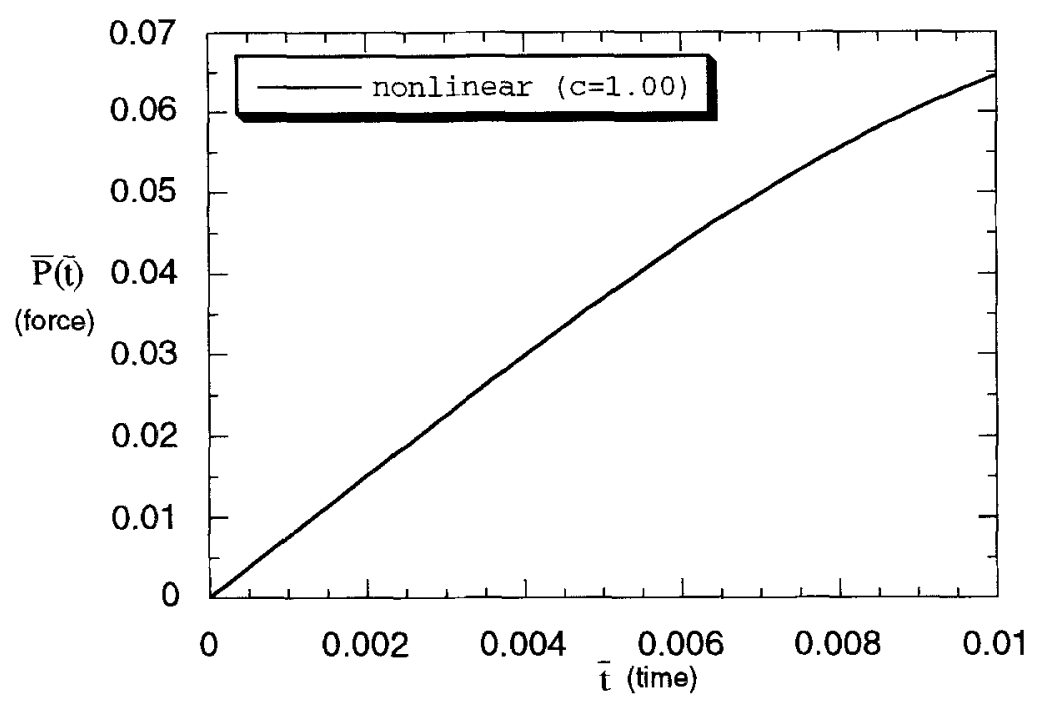

Fig. 10. Force history for the cantilever beam under deflection control

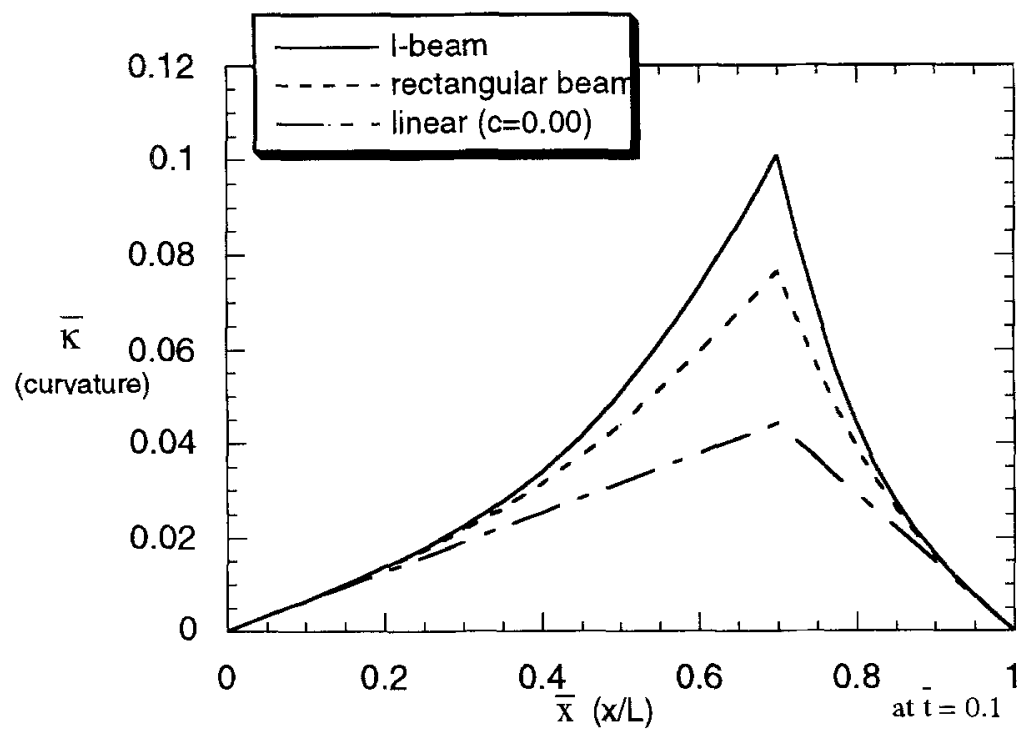

Fig. 11. Curvature distributions along a simply supported beam for the rectangular and $I$-shaped sections, at the final simulation time $\bar{t}=0.1$

$\bar{t}=0.04,0.08$, and 0.10 . At $\bar{t}=0.08$, there has been little acceleration of curvature creep for the rectangular section, while there has been a substantial amount for the $I$-section. The region of large curvature grows faster for the $I$-section.

The NLVE and LVE deflections along the beam with time are illustrated in Fig. 13, for the $I$ shaped section. The corresonding results for the rectangular section are similar but smaller. They are omitted for the sake of brevity and may be found in [14]. The NLVE deflection is close to the LVE deflection up to $\bar{t}=0.04$. For later times, the deviation grows rapidly. Also, up to $\bar{t}=0.08$, the deflections for the two cross sections are very close. The deflection for the $I$-section grows faster and is $20 \%$ bigger than for the rectangular section at the final 


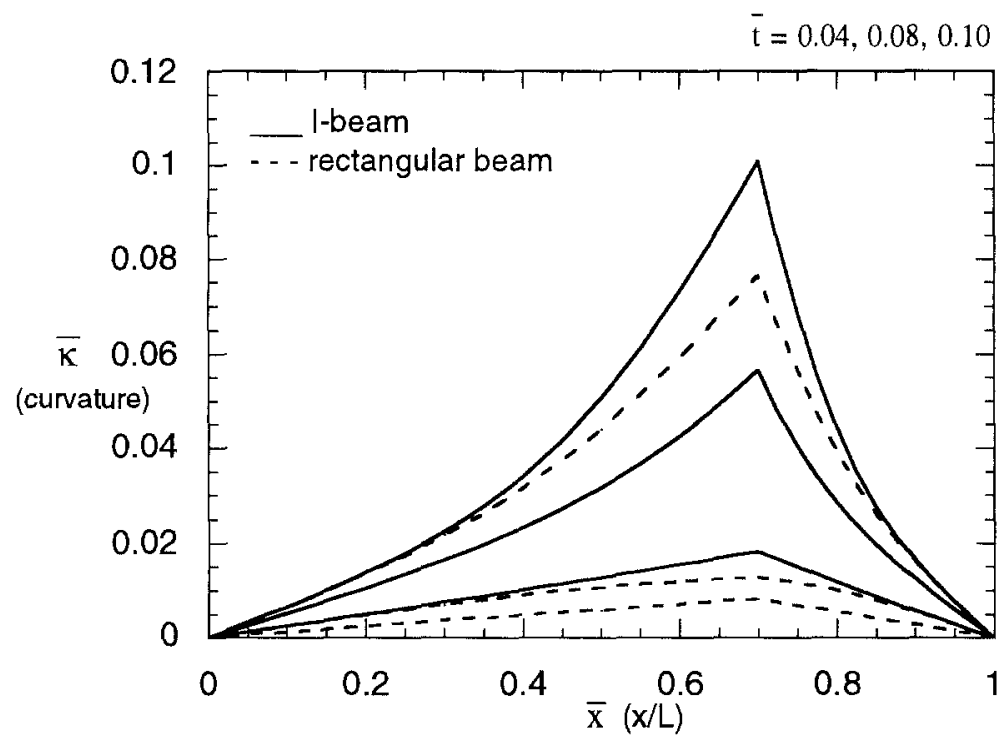

Fig. 12. Curvature distributions along a simply supported beam for the rectangular and $I$-shaped sections, for various times

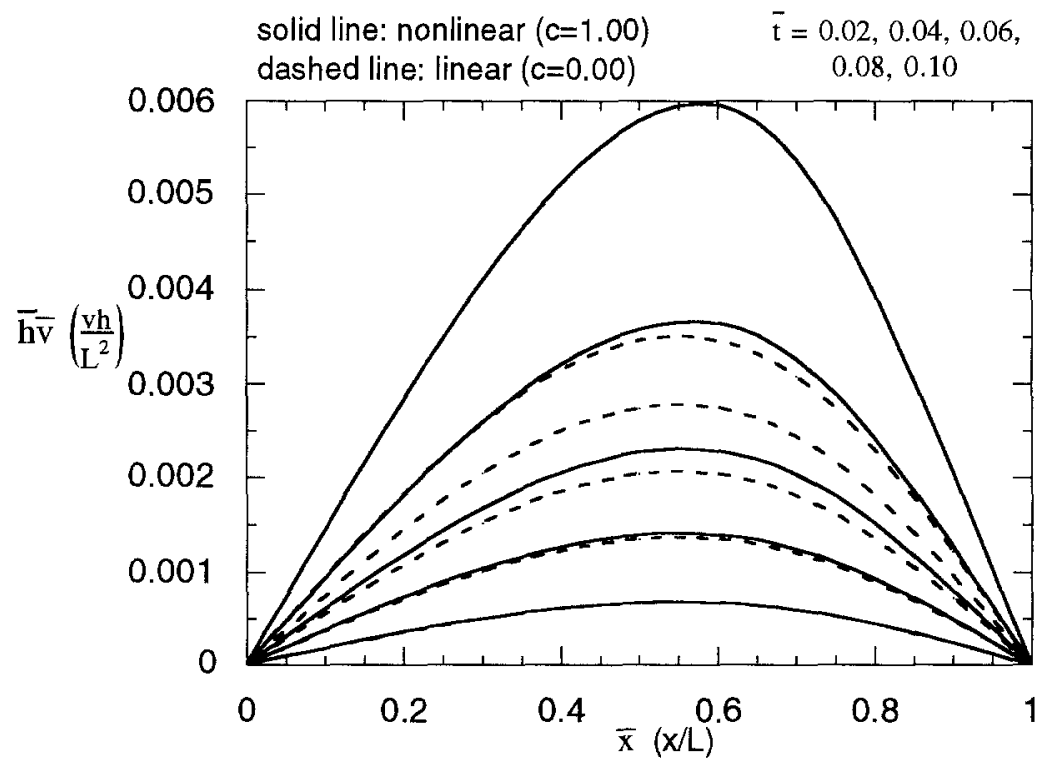

Fig. 13. Deflection distributions along the simply supported beam for the $I$-shaped section for various times

simulation time $\bar{t}=0.1$. Another phenomenon associated with the acceleration of stressrelaxation is the time dependent location of the point of maximum deflection. For the LVE case, the location of maximum delfection is the same at all times. For the NLVE case, the location moves from $\bar{x}=0.55$ to $\bar{x} \approx 0.575$ at $\bar{t}=0.1$ for both cross sections.

The NLVE and LVE stresses at the top element along the beam length at the final simulation time $\bar{t}=0.1$ are shown in Fig. 14. For LVE case, the stress profiles for the $I$-section and the rectangular section coincide. However, for the NLVE case, the profile depends on the 


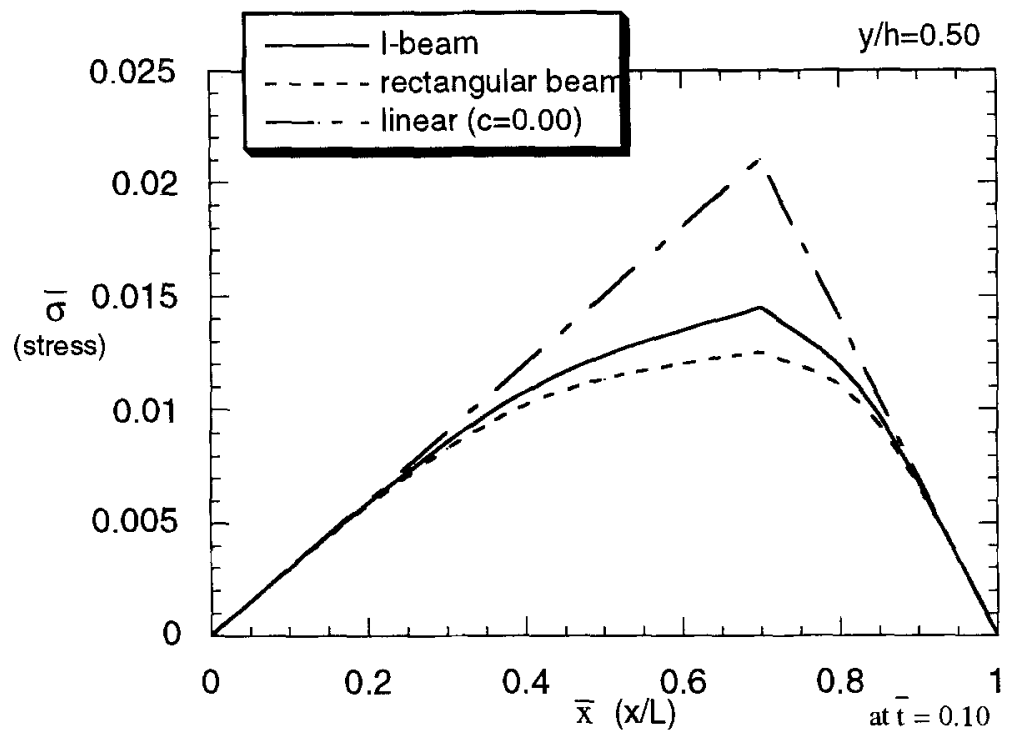

Fig. 14. Stress distribution at the top elements of the simply supported beam, for the rectangular and $I$ shaped sections, at the final simulation time $\bar{t}=0.1$

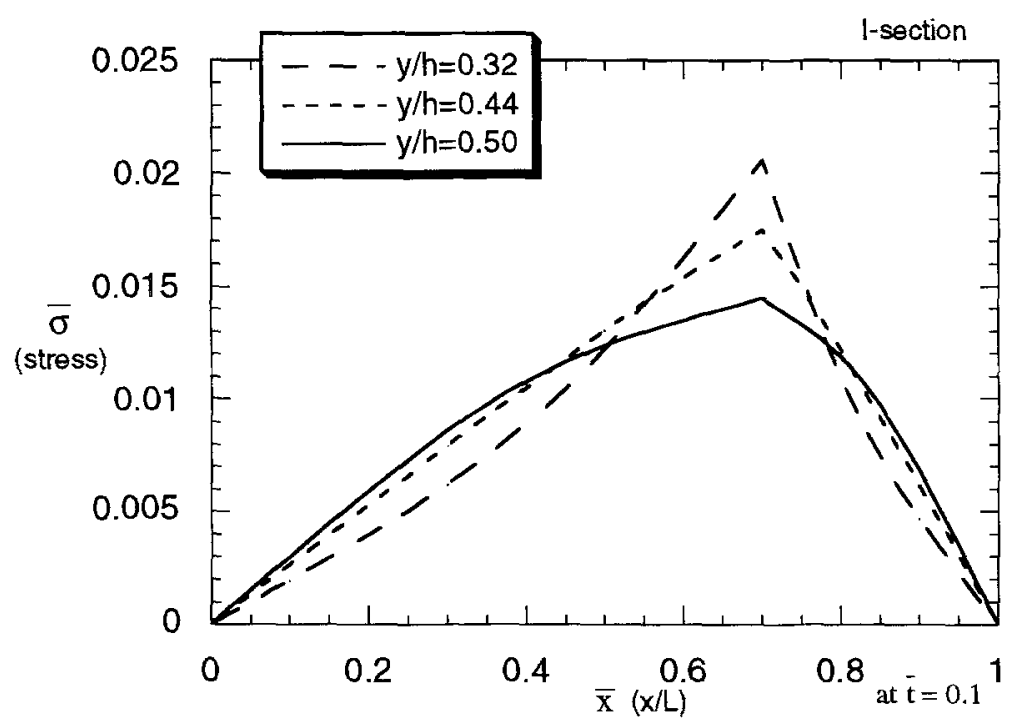

Fig. 15. Stress distributions along the simply supported beam for the various positions through the beam thickness, for the $I$-shaped section, at the final simulation time $\bar{t}=0.1$

shape of the cross section. It can be seen that the stress profiles for the $I$-shaped and rectangular cross sections are similar, but the $I$-section has higher stress. The NLVE stresses at material elements at $\bar{y}=0.32,0.44$, and 0.50 along the beam at the final simulation time $\bar{t}=0.1$ are shown in Fig. 15 for the $I$-shaped section. The corresponding results for the rectangular section are omitted for the sake of brevity and may be found in [14]. For $0 \leq \bar{x} \leq 0.4$ and $0.85 \leq \bar{x} \leq 1.00$, the stress increases with distance from the centroid to the top of the $I$-section. For $0.4 \leq \bar{x} \leq 0.85$, the element with maximum stress is close to $\bar{y}=0.32$. This shows that the material nonlinearity is small near the ends and has major influence in the neighborhood of the applied force. 


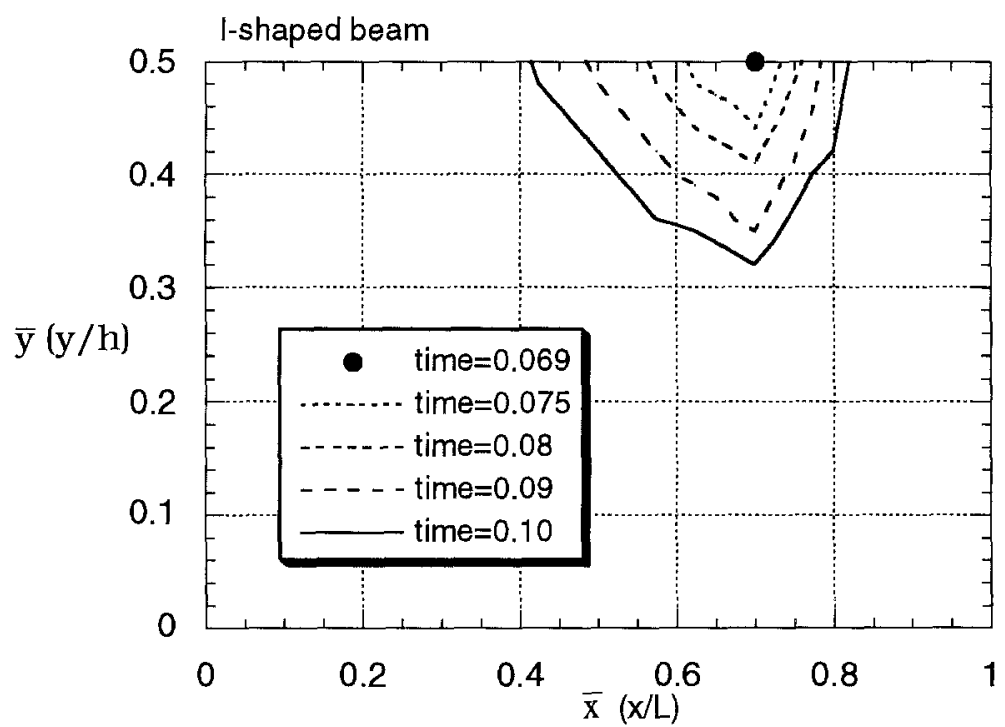

Fig. 16. Growth of stress relaxation zones in a simply supported beam for the $I$-shaped section

Zones of the acceleration of stress relaxation for the simply supported beam were determined in the same manner as for the cantilever beam. The propagation of these zones for the $I$-shaped section is illustrated in Fig. 16. The heavy dot at $\bar{t}=0.0690$ denotes the time of onset and the position of the stress relaxation zone. The time of onset for the rectangular section $\bar{t}=0.0706$. The stress relaxation zone for rectangular section is similar and is omitted for the sake of brevity (see [14]).

\section{Conclusion}

The results presented here show a phenomenon arising from the interaction of the acceleration of stress relaxation with strain and the spatial variation of strain, within the structural theory of beams. The regions with the greatest bending moments and stresses, within the context of the linear theory, become regions of accelerated stress relaxation when the material response is described by the constitutive equation with the "strain clock". There is a time after transverse loads are applied to the beam when the stresses at the outer material elements in these cross sections begin to decrease, the location of the maximum stress begins to move to the interior of the cross section and the curvature begins to increase rapidly. These events have been described as the onset and growth of a "viscoelastic hinge".

The occurrence of this viscoelastic hinge in a specific beam will depend on the properties of the polymer, the geometry of the beam and the loading conditions. An accounting for this hinge can be an important part of the design of polymeric beams which operate near yield. If the beam is designed using linear viscoelasticity, there can be some time after it is put into service when the beam may fail to carry the design load or may undergo unacceptably large displacements. Conversely, there may be an application in which it is desirable to impose a large curvature. The hinge effect shows that this can be done rapidly and with reduced loads. 


\section{References}

[1] Shay, R. M. Jr., Caruthers, J. M.: A new nonlinear viscoelastic constitutive equation for predicting yield in amorphous solid polymers. J. Rheology 30, $781-827$ (1986).

[2] Knauss, W. G., Emri, I. J.: Nonlinear viscoelasticity based on free volume consideration. Comput. Struct. 13, 123-128 (1981).

[3] Knauss, W. G., Emri, I. J.: Volume change and the nonlinear thermo-viscoelastic constitution of polymers. Polymer Engng. Sci. 27, 86-100 (1987).

[4] Wineman, A. S., Waldron, W. K. Jr.: Interaction of nonhomogeneous shear, nonlinear viscoelasticity and yield of a solid polymer. Polymer Engng. Sci. 33, 1217-1228 (1993).

[5] Wineman, A. S., Min, J. H.: Biaxial yield for nonlinearly viscoelastic material with a strain clock. Mechanics of Time-Dependent Materials 2, 37-58 (1998).

[6] McKenna, G. B., Zapas, L. J.: Nonlinear viscoelastic behavior of poly(methylmethacrylate) in torsion. J. Rheology 23, 151-166 (1979).

[7] Moran, B., Knauss, W. G.: Crack-tip stress and deformation fields in strain softening nonlinearly viscoelastic materials. J. Appl. Mech. 59, 95-101 (1992).

[8] Wineman, A. S., Waldron, W. K. Jr.: Shear and normal stress effects in finite circular shear of a compressible nonlinear viscoelastic solid. J. Non-linear Mech. 31, 345-369 (1996).

[9] Wineman, A. S., Kolberg, R.: Response of beams of nonlinear viscoelastic materials exhibiting strain dependent stress relaxation. Int. J. Non-linear Mech. 32, 863-883 (1997).

[10] Wineman, A. S., Min, J. H.: The pressurized cylinder problem for nonlinear viscoelastic materials with a strain clock. Math. Mech. Solids 1, 393-410 (1996).

[11] Wineman, A. S., Min, J. H.: The pressurized sphere problem for nonlinear viscoelastic materials with a strain clock. Trans. of the Canadian Society for Mech. Engng 20, 217-232 (1996).

[12] Tschoegl, N. W.: The phenomenological theory of linear viscoelastic behavior, pp. 528-532. Berlin: Springer 1989.

[13] Staverman, A. J., Schwarzl, F.: Linear deformation behavior of high polymers. In: Die Physik der Hochpolymeren, vol. 4 (Stuart, H. A., ed.) pp. 1-125. Berlin: Springer 1956.

[14] Min, J. H.: Dilatation enhanced stress relaxation effects in nonlinear viscoelastic solid polymeric structures. Ph.D.thesis, University of Michigan, Ann Arbor (1995).

Authors' address: A. Wineman and J. H. Min, Department of Mechanical Engineering and Applied Mechanics, University of Michigan, Ann Arbor, Michigan, 48109, USA 